\title{
ON A NONLINEAR PARTIAL DIFFERENTIAL EQUATION ARISING IN MAGNETIC RESONANCE ELECTRICAL IMPEDANCE TOMOGRAPHY
}

\author{
SUNGWHAN KIM $\dagger$, OHIN KWON $\ddagger$, JIN KEUN SEO †, AND JEONG-ROCK YOON $\S$
}

\begin{abstract}
This paper considers the fundamental questions, such as existence and uniqueness, of a mathematical model arising in MREIT system, which is electrical impedance tomography technique integrated with magnetic resonance imaging. The mathematical model for MREIT is the Neumann problem of a nonlinear elliptic partial differential equation $\nabla \cdot\left(\frac{a(x)}{|\nabla u(x)|} \nabla u(x)\right)=0$. We show that this Neumann problem belongs to one of two cases: either infinitely many solutions or no solution exist. This explains rigorously the reason why we have used the modified model in [7] which is a system of the Neumann problem associated with two different Neumann data. For this modified system, we prove a uniqueness result on the edge detection of a piecewise continuous conductivity distribution.
\end{abstract}

\section{IntRoduction}

Magnetic resonance electrical impedance tomography(MREIT) is a new imaging technique of reconstructing the cross-sectional conductivity distribution of a human body by means of the electrical impedance tomography(EIT) technique integrated with the magnetic resonance imaging(MRI) technique. EIT technique to estimate the conductivity distribution uses data obtained by injecting a known current into the body through electrodes placed on the surface and measuring the resulting voltage difference recorded on the electrodes. EIT problem is known as a highly illposed inverse problem due to its low sensitivity of data to the change in conductivity value. (See [14].) MREIT is designed to overcome this severe ill-posedness of EIT problem by making good use of a recent MRI technique, so called current density imaging (CDI) of measuring the internal current density distribution. (See for related works $[4,6,10,11,12,15]$.)

In the recent paper [7], a new reconstruction algorithm for MREIT was developed to provide a high resolution conductivity image. This algorithm is based on a new mathematical modeling which is involved with a nonlinear partial differential equation instead of the linear conductivity equation. Although the algorithm has achieved successful numerical results in simulations, there has been no related mathematical theory for the new model such as existence and uniqueness. This paper is intended to provide answers to those questions.

Let us explain the mathematical model for MREIT which has been introduced in [7]. Let the cross-section of the cylindrical body occupy a bounded domain $\Omega \subset \mathbb{R}^{2}$. When a current is injected transversely through the outer surface of the body, it induces an electrical potential distribution $u$ that satisfies the two-dimensional conductivity equation

$$
\nabla \cdot(\sigma \nabla u)=0 \quad \text { in } \Omega
$$

$\dagger$ Department of Mathematics, Yonsei University, Seoul 120-749, Korea.

$\ddagger$ Department of Mathematics and Natural Science Research Institute, Yonsei University, Seoul $120-749$, Korea.

$\S$ School of Mathematics, Korea Institute of Advanced Study, Seoul 130-012, Korea. 


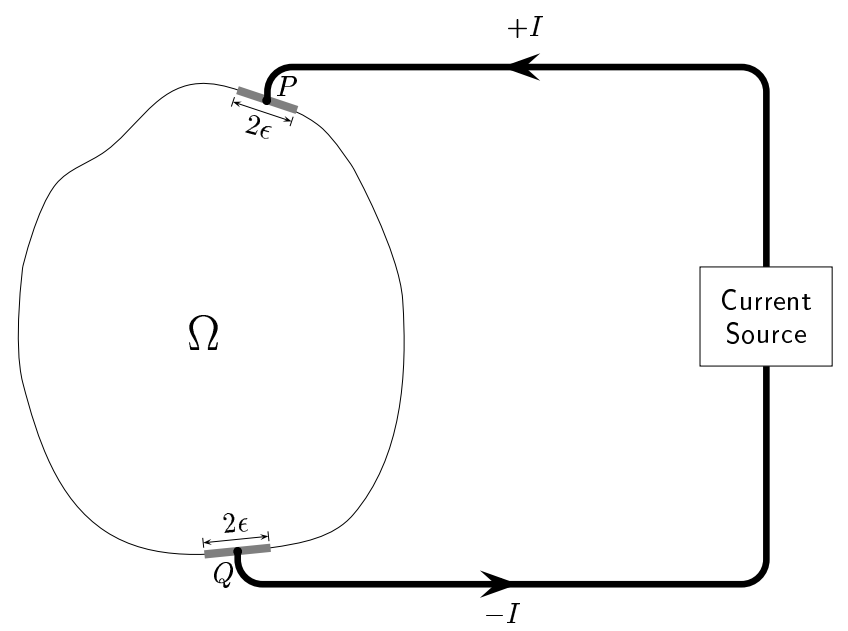

FiguRE 1. An illustration for the model where the current $I$ is applied through a pair of electrodes attached on the boundary.

where $\sigma$ denotes the conductivity coefficient of the body which we want to reconstruct. This unknown two variable function $\sigma$ may be regarded as a piecewise continuous positive function. In the MREIT model, the current is applied through a pair of electrodes attached on the boundary $\partial \Omega$ : If both electrodes of width $2 \epsilon$ are attached at points $P, Q \in \partial \Omega$, respectively, then the current density on the boundary can be approximated by a function

$$
g(x)= \begin{cases}+\frac{I}{2 \epsilon} & \text { on }\{|x-P|<\epsilon\} \cap \partial \Omega, \\ -\frac{I}{2 \epsilon} & \text { on }\{|x-Q|<\epsilon\} \cap \partial \Omega, \\ 0 & \text { otherwise, }\end{cases}
$$

where $I$ is the current sent to both electrodes at $P$ and $Q$, the illustration of which is shown in Figure 1. For more details, see the ave-gab model in $[3,9]$.

With this current $g$, the resulting internal current density vector $\mathbf{J}=-\sigma \nabla u$ is divergence-free and satisfies the boundary condition

$$
\sigma \frac{\partial u}{\partial \nu}=-\mathbf{J} \cdot \nu=g \quad \text { on } \partial \Omega
$$

where $\nu$ denotes the outward unit normal vector to $\partial \Omega$. Moreover, the MREIT system furnishes the internal data $a=|\mathbf{J}|=\sigma|\nabla u|$ which is measured and processed in MRI system $[7,15]$. We want to utilize this acquisition data $a$ by substituting

$$
\sigma(x)=\frac{a(x)}{|\nabla u(x)|}, \quad x \in \Omega,
$$

into the conductivity equation (1.1) and the Neumann boundary condition (1.3). As a result, the linear boundary value problem (1.1) and (1.3) with two unknowns $\sigma$ and $u$ is reduced to the following nonlinear Neumann boundary value problem with one unknown $u$,

$$
\begin{gathered}
\nabla \cdot\left(\frac{a}{|\nabla u|} \nabla u\right)=0 \quad \text { in } \Omega \\
\frac{a}{|\nabla u|} \frac{\partial u}{\partial \nu}=g \quad \text { on } \partial \Omega, \quad \text { and } \int_{\partial \Omega} u d s=0
\end{gathered}
$$


where the last condition means the potential reference condition. To be precise, the electric potential $u \in H^{1}(\Omega)$ can be viewed as a weak solution satisfying

$$
\int_{\Omega} \frac{a}{|\nabla u|} \nabla u \cdot \nabla \phi d x=\int_{\partial \Omega} g \phi d s \quad \text { for all } \quad \phi \in H^{1}(\Omega)
$$

with a constraint $\int_{\partial \Omega} u d s=0$.

It is natural to investigate the fundamental mathematical issue of the nonlinear boundary value problem (1.5), such as existence and uniqueness. In practice, the existence may not be a serious problem but the uniqueness must be seriously taken into account. In case when we have not unique but several different solutions, there will be several corresponding distinct conductivity images and we cannot judge which one would be the actual image.

Unfortunately, in section 3 we will prove that once the problem (1.5) has a solution, then it always has infinitely many solutions under a practically acceptable assumption that will be precisely defined in section 3. Hence the model (1.5) using one measurement is insufficient for the reconstruction of the conductivity distribution. A numerical example is presented in section 5 to show how different conductivity images can be reconstructed with the same data $(a, g)$. Moreover, we also prove in section 3 that the problem (1.5) in general does not have existence result even if $a$ is smooth. We think that the existence of the solution to the problem (1.5) is related to some complicated connection between $a$ and $g$, because the internal current density $a$ depends on the choice of the injected current $g$.

Thus the model should be modified in order to guarantee the uniqueness. In section 4, we apply two different currents $g_{1}$ and $g_{2}$ approximated in the same manner as in (1.2) attaching two different pairs of electrodes $\left\{P_{1}, Q_{1}\right\}$ and $\left\{P_{2}, Q_{2}\right\}$. Since the conductivity distribution $\sigma$ is independent of the change of injected currents, from the relation (1.4) we may assume

$$
\frac{a_{1}(x)}{\left|\nabla u_{1}(x)\right|}=\frac{a_{2}(x)}{\left|\nabla u_{2}(x)\right|}, \quad x \in \Omega,
$$

where $u_{j}$ is a solution to the nonlinear Neumann boundary value problem (1.5) when $g$ and $a$ are replaced by $g_{j}$ and $a_{j}(j=1,2)$. This leads to the following nonstandard system of equations

$$
\begin{gathered}
\nabla \cdot\left(\frac{a_{j}}{\left|\nabla u_{j}\right|} \nabla u_{j}\right)=0 \quad \text { in } \Omega, \\
\frac{a_{1}}{\left|\nabla u_{1}\right|}=\frac{a_{2}}{\left|\nabla u_{2}\right|} \quad \text { in } \Omega, \\
\frac{a_{j}}{\left|\nabla u_{j}\right|} \frac{\partial u_{j}}{\partial \nu}=g_{j} \quad \text { on } \partial \Omega, \\
\int_{\partial \Omega} u_{j} d s=0,
\end{gathered}
$$

for $j=1,2$. With this modified model and a practically acceptable assumption, in section 4 we are able to establish an important uniqueness result which may look strange at a glance.

In the following section 2 , we define a space for physically meaningful conductivity distributions and recall some regularity properties of elliptic partial differential equations for further usage.

\section{Definitions and Preliminary}

We assume that $\Omega \subset \mathbb{R}^{2}$, a cross-section of the human body, is a simply connected bounded domain with $\mathcal{C}^{2}$ boundary. The conductivity distribution $\sigma$ on the crosssection $\Omega$ may be regarded as a piecewise continuous function because distinct 
tissues have different conductivities. So, we may assume that $\sigma$ belongs to the following class

$$
\begin{array}{r}
\Sigma:=\left\{\sigma=\sigma_{0}+\sum_{k=1}^{M} \sigma_{k} \chi_{D_{k}} \mid M \in \mathbb{N}, 0<\sigma<\infty, \bar{D}_{k} \subset \Omega, \bar{D}_{k} \cap \bar{D}_{\ell}=\emptyset \text { for } k \neq \ell,\right. \\
\left.\sigma_{0} \in \mathcal{C}^{\alpha}(\bar{\Omega}), \sigma_{k} \in \mathcal{C}^{\alpha}\left(\bar{D}_{k}\right), \sigma_{k} \neq 0 \text { on } \partial D_{k}, \partial D_{k} \text { is a } \mathcal{C}^{2} \text { boundary }\right\}
\end{array}
$$

where $\chi_{D_{k}}$ denotes the characteristic function for $D_{k}$ and $0<\alpha<1$ is not an important number. With this setting, for any $\sigma=\sigma_{0}+\sum_{k=1}^{M} \sigma_{k} \chi_{D_{k}} \in \Sigma$, we easily see that

$$
\begin{gathered}
\sigma \in \mathcal{C}^{\alpha}\left(\cup_{k=1}^{M} \bar{D}_{k}\right) \cap \mathcal{C}^{\alpha}\left(\Omega \backslash \cup_{k=1}^{M} D_{k}\right), \\
\{x \in \Omega \mid \sigma \text { is discontinuous at } x\}=\bigcup_{k=1}^{M} \partial D_{k} .
\end{gathered}
$$

For a given current $g$ in (1.2) and $\sigma=\sigma_{0}+\sum_{k=1}^{M} \sigma_{k} \chi_{D_{k}} \in \Sigma$, let $u$ be the solution of the classical Neumann boundary value problem

$$
\begin{gathered}
\nabla \cdot(\sigma \nabla u)=0 \quad \text { in } \Omega, \\
\sigma \frac{\partial u}{\partial \nu}=g \quad \text { on } \partial \Omega, \quad \text { and } \int_{\partial \Omega} u d s=0 .
\end{gathered}
$$

From the basic theory of elliptic partial differential equation $[5,8]$, we know

$$
\begin{aligned}
& \text { (a) } u \in \mathcal{C}(\bar{\Omega}), \\
& \text { (b) } \nabla u \in \mathcal{C}^{\alpha}\left(\cup_{k=1}^{M} \bar{D}_{k}\right) \cap \mathcal{C}^{\alpha}\left(\Omega \backslash \cup_{k=1}^{M} D_{k}\right), \\
& \text { (c) } \sigma_{0}(\xi) \nabla u^{+}(\xi) \cdot \nu(\xi)=\left(\sigma_{0}(\xi)+\sigma_{k}(\xi)\right) \nabla u^{-}(\xi) \cdot \nu(\xi) \text { if } \xi \in \partial D_{k}, \\
& \text { (d) } \nabla u^{+}(\xi) \cdot \tau(\xi)=\nabla u^{-}(\xi) \cdot \tau(\xi) \text { if } \xi \in \partial D_{k},
\end{aligned}
$$

where $\nu$ and $\tau$ are the outward unit normal vector and the unit tangent vector to $\partial D_{k}$, respectively, and $u^{+}, u^{-}$are defined by

$$
u^{+}=\left.u\right|_{\Omega \backslash \bigcup_{k=1}^{M} \bar{D}_{k}} \quad \text { and } \quad u^{-}=\left.u\right|_{\bigcup_{k=1}^{M} D_{k}} .
$$

Moreover, owing to the choice of $g$ as in (1.2), we can show that

$$
\nabla u(x) \neq 0 \quad \text { for all } x \in \Omega,
$$

the proof of which can be found in $[1,2,13]$. Indeed, (2.5) holds if nonzero $g$ satisfies the following condition; there exist two disjoint $\operatorname{arcs} \Gamma^{+}$and $\Gamma^{-}$contained in $\partial \Omega$ such that

$$
\Gamma^{+} \cup \Gamma^{-}=\partial \Omega, \quad \text { and } \quad \Gamma^{+} \subset\{g \geq 0\}, \quad \Gamma^{-} \subset\{g \leq 0\},
$$

the detailed proof of which will be given in Remark 4.2 for completeness.

\section{Non-existence And Non-Uniqueness}

In this section, we will prove that the nonlinear Neumann boundary value problem (1.5) under a practically acceptable assumption is in general not uniquely solvable by constructing infinitely many different solutions from one solution and by giving an example for non-existence.

From the relation (1.4) between the conductivity distribution $\sigma$ and the measured current density $a$, we may assume that a practically meaningful solution $u$ of the Neumann problem (1.5) satisfies

$$
\frac{a(x)}{|\nabla u(x)|} \in \Sigma,
$$


since $\Sigma$ contains almost all cases of piecewise continuous conductivities that may happen in the real situation. So, the practical solution $u$ can be considered as a $H^{1}(\Omega)$ solution of the more complicated problem where $g$ is given as in (1.2),

$$
\begin{gathered}
\nabla \cdot\left(\frac{a}{|\nabla u|} \nabla u\right)=0 \quad \text { in } \Omega, \quad \frac{a}{|\nabla u|} \in \Sigma, \\
\frac{a}{|\nabla u|} \frac{\partial u}{\partial \nu}=g \quad \text { on } \partial \Omega, \quad \text { and } \int_{\partial \Omega} u d s=0 .
\end{gathered}
$$

Hence, if $u$ is a solution of (3.2), it satisfies (2.5) and the properties $(a)-(d)$ in (2.4). By the property (b) in (2.4) and (3.1), $a=\frac{a}{\mid \nabla u}|\nabla u|$ must be also a piecewise continuous function in $\Omega$.

We can easily construct a solution for problem (3.2): For any $\sigma \in \Sigma$, there exists a unique solution $u_{\sigma}$ to the classical Neumann problem (2.3), and this $u_{\sigma}$ is also a solution to (3.2) when $a$ is given by $a=\sigma\left|\nabla u_{\sigma}\right|$. To our surprise, the problem (3.2) with this $a$ has infinitely many solutions and $u_{\sigma}$ is just one of them. The following theorem states this non-uniqueness result.

Theorem 3.1. If the nonlinear problem (3.2) has a solution, then it has infinitely many solutions.

Proof. Suppose $u$ is a solution of the problem (3.2). We will construct infinitely many solutions by means of $u$. Since $u$ satisfies the property (a) in (2.4) and (2.5), we have $\min _{x \in \bar{\Omega}} u(x)<\max _{x \in \bar{\Omega}} u(x)$. For any $t \in\left(\min _{\bar{\Omega}} u, \max _{\bar{\Omega}} u\right)$ and $\lambda>0$, we define

$$
u_{t, \lambda}:= \begin{cases}u+c & \text { in } \Omega_{t}^{+}, \\ \lambda u+(1-\lambda) t+c & \text { in } \Omega_{t}^{-},\end{cases}
$$

where the number $c$ is chosen so that $\int_{\partial \Omega} u_{t, \lambda} d s=0$ and

$$
\Omega_{t}^{+}:=\{x \in \Omega \mid u(x) \geq t\} \quad \text { and } \quad \Omega_{t}^{-}:=\{x \in \Omega \mid u(x)<t\} .
$$

Then it is easy to see that $u_{t, \lambda} \in \mathcal{C}(\bar{\Omega})$ and

$$
\frac{\nabla u_{t, \lambda}(x)}{\left|\nabla u_{t, \lambda}(x)\right|}=\frac{\nabla u(x)}{|\nabla u(x)|} \quad \text { for all } x \in \Omega .
$$

Since the possible discontinuity regions of $a /\left|\nabla u_{t, \lambda}\right|$ are $\{x \in \Omega \mid u(x)=t\}$ and those of $a /|\nabla u|$, we easily verify that $a /\left|\nabla u_{t, \lambda}\right| \in \Sigma$. Therefore $u_{t, \lambda}$ is also a solution to (3.2), which completes the proof.

In section 5, we will present two distinct (numerically obtained) solutions that will arise in the complicated real situation, which solve the same problem (3.2).

Now we investigate the existence question. For simplicity, we confine ourselves to a unit square domain $\Omega=(0,1) \times(0,1)$ in $\mathbb{R}^{2}$. Let $x=\left(x_{1}, x_{2}\right)$ denote a point in $\Omega$ and the current pattern $g$ on $\partial \Omega$ be given by

$$
g(x)= \begin{cases}-1 & \text { if } x_{1}=0 \\ 1 & \text { if } x_{1}=1 \\ 0 & \text { otherwise. }\end{cases}
$$

The next theorem furnishes an example for the non-existence of the problem (3.2).

Theorem 3.2. Let $\Omega=(0,1) \times(0,1)$ and $g$ be given in (3.3). Assume that $a$ in (3.2) depends only on $x_{1}-$ variable, that is, $a\left(x_{1}, x_{2}\right)=a\left(x_{1}\right)$. The necessary and sufficient condition for the existence of solution to the problem (3.2) is a $\equiv 1$.

Proof. If $a \equiv 1$, then clearly $u(x)=x_{1}-\frac{1}{2}$ is a solution of the problem (3.2) which would be one of the infinitely many solutions. This proves the sufficiency. 
To show the necessity, suppose that the problem (3.2) has a solution $u$. First, we will prove that $a(t) \geq 1$ for all $t \in(0,1)$. For convenience, we denote

$$
\begin{array}{ll}
l_{1}=\left\{x \in \partial \Omega \mid x_{2}=0\right\}, & l_{2}=\left\{x \in \partial \Omega \mid x_{1}=1\right\}, \\
l_{3}=\left\{x \in \partial \Omega \mid x_{2}=1\right\}, & l_{4}=\left\{x \in \partial \Omega \mid x_{1}=0\right\},
\end{array}
$$

and $R_{t}:=\left\{x \in \Omega \mid 0<x_{1}<t\right\}$ be a rectangle on the left side of the line $\left\{x_{1}=t\right\}$.

Applying the divergence theorem on $R_{t}$, we obtain

$$
\begin{aligned}
0 & =\int_{\partial R_{t}} \frac{a}{|\nabla u|} \frac{\partial u}{\partial \nu} d s \\
& =\int_{\partial R_{t} \cap \partial \Omega} g d s+\int_{\partial R_{t} \cap\left\{x_{1}=t\right\}} \frac{a}{|\nabla u|} \nabla u \cdot \nu d s \\
& =-\int_{l_{4}} d s+a(t) \int_{\partial R_{t} \cap\left\{x_{1}=t\right\}} \frac{\nabla u \cdot \nu}{|\nabla u|} d s \\
& \leq-1+a(t),
\end{aligned}
$$

since $a(x)=a(t)$ on $\partial R_{t} \cap\left\{x_{1}=t\right\}$ and $\nabla u \cdot \nu \leq|\nabla u|$ where $\nu$ denotes the outward unit normal to $R_{t}$. Hence we have $a(t) \geq 1$ for all $t \in(0,1)$.

Now, we will show that the level curve $\Gamma_{t}:=\{x \in \Omega \mid u(x)=u(t, 0)\}$ is the vertical line $\left\{x \in \Omega \mid x_{1}=t\right\}$ for all $t \in(0,1)$. Since $a \geq 1$, the choice of $g$ in (3.3) and the Neumann boundary condition in (3.2) yield $\partial u / \partial \nu(t, 0)=0$, which implies

$$
\min _{x \in \bar{\Omega}} u(x)<u(t, 0)<\max _{x \in \bar{\Omega}} u(x) .
$$

Thus $\Omega_{t}:=\{x \in \Omega \mid u(x)<u(t, 0)\}$ is a nonempty open subset of $\Omega$ and $(t, 0) \in \partial \Omega_{t}$. It is easy to see that

$$
\mathcal{H}^{1}\left(\partial \Omega_{t} \cap l_{4}\right)<\mathcal{H}^{1}\left(\partial \Omega_{t} \backslash\left(l_{1} \cup l_{3} \cup l_{4}\right)\right) \quad \text { if } \Gamma_{t} \neq\left\{x \in \Omega \mid x_{1}=t\right\},
$$

where $\mathcal{H}^{1}(L)$ denotes the arclength of the curve $L$.

Applying the divergence theorem on $\Omega_{t}$, we have

$$
\begin{aligned}
0 & =\int_{\partial \Omega_{t}} \frac{a}{|\nabla u|} \frac{\partial u}{\partial \nu} d s \\
& =\int_{\partial \Omega_{t} \cap \partial \Omega} g d s+\int_{\partial \Omega_{t} \backslash \partial \Omega} \frac{a}{|\nabla u|} \frac{\partial u}{\partial \nu} d s \\
& =-\mathcal{H}^{1}\left(\partial \Omega_{t} \cap l_{4}\right)+\mathcal{H}^{1}\left(\partial \Omega_{t} \cap l_{2}\right)+\int_{\partial \Omega_{t} \backslash \partial \Omega} a \frac{\nabla u \cdot \nu}{|\nabla u|} d s,
\end{aligned}
$$

where $\nu$ denotes the outward unit normal to $\Omega_{t}$. Since $u(x)<u(t, 0)$ in $\Omega_{t}$ and $u(x)=u(t, 0)$ on $\partial \Omega_{t} \backslash \partial \Omega$, we have $\nu=\nabla u /|\nabla u|$ on $\partial \Omega_{t} \backslash \partial \Omega$, which implies

$$
\frac{\nabla u \cdot \nu}{|\nabla u|}=1 \quad \text { on } \partial \Omega_{t} \backslash \partial \Omega \text {. }
$$

By the above identity and the fact that $a \geq 1$, from (3.6) we get

$$
\begin{aligned}
\mathcal{H}^{1}\left(\partial \Omega_{t} \cap l_{4}\right) & =\mathcal{H}^{1}\left(\partial \Omega_{t} \cap l_{2}\right)+\int_{\partial \Omega_{t} \backslash \partial \Omega} a d s \\
& \geq \mathcal{H}^{1}\left(\partial \Omega_{t} \cap l_{2}\right)+\mathcal{H}^{1}\left(\partial \Omega_{t} \backslash \partial \Omega\right) \\
& =\mathcal{H}^{1}\left(\partial \Omega_{t} \backslash\left(l_{1} \cup l_{3} \cup l_{4}\right)\right) .
\end{aligned}
$$

Hence, from (3.5) it must be $\Gamma_{t}=\left\{x \in \Omega \mid x_{1}=t\right\}$, that is, $u\left(t, x_{2}\right)=u(t, 0)$ for $0<x_{2}<1$, which implies $(\nabla u \cdot \nu) /|\nabla u|= \pm 1$ on $\partial R_{t} \cap\left\{x_{1}=t\right\}$ in (3.4). Thus from (3.4), we have

$$
0=\int_{\partial R_{t}} \frac{a}{|\nabla u|} \frac{\partial u}{\partial \nu} d s=-1 \pm a(t)
$$


By the knowledge of $a \geq 1$, we conclude that $a(t)=1$ for all $t \in(0,1)$, which proves the necessity.

\section{UNIQUENESS OF EDGE IN A MODIFIED SYSTEM}

In order not to go astray from the main point of MREIT, we must focus on the final goal of MREIT which aims to reconstruct the conductivity image $\sigma$. In section 3 , we have observed that the model (3.2) may have infinitely many solutions $u$, so has infinitely many distinct conductivity images $\sigma=a /|\nabla u|$. Thus, the model (3.2) is not appropriate for making reconstruction algorithm. This is the main reason why the modified system (1.6) has been introduced in [7] for the reconstruction algorithm that has been successfully demonstrated to provide accurate high resolution conductivity images.

Although, in numerical simulations in [7], the system (1.6) seems to have uniqueness, we are not able to prove the uniqueness rigorously for the present, but we could prove a practically useful uniqueness result which guarantees the unique detection of the edges of the conductivity image. This means that the system (1.6) uniquely determines the interface where the conductivity distribution $\sigma$ is discontinuous.

As discussed at the beginning of section 3 , if we assume that $\left(u_{1}, u_{2}\right)$ is a practically acceptable solution of the system (1.6), we may impose the assumption

$$
\sigma=\frac{a_{1}}{\left|\nabla u_{1}\right|}=\frac{a_{2}}{\left|\nabla u_{2}\right|} \in \Sigma
$$

which is the two measurement analogue of the assumption (3.1). By plugging this assumption into the system (1.6), we have the following system that will be considered in this section,

$$
\begin{gathered}
\nabla \cdot\left(\frac{a_{j}}{\left|\nabla u_{j}\right|} \nabla u_{j}\right)=0 \quad \text { in } \Omega, \\
\frac{a_{1}}{\left|\nabla u_{1}\right|}=\frac{a_{2}}{\left|\nabla u_{2}\right|} \in \Sigma, \\
\frac{a_{j}}{\left|\nabla u_{j}\right|} \frac{\partial u_{j}}{\partial \nu}=g_{j} \quad \text { on } \partial \Omega \\
\int_{\partial \Omega} u_{j} d s=0
\end{gathered}
$$

for $j=1,2$. For the uniqueness of $(4.1)$, we need to choose an appropriate pair of current patterns $g_{1}$ and $g_{2}$ to have

$$
\left|\nabla u_{1}(x) \times \nabla u_{2}(x)\right|>0 \text { for all } x \in \Omega .
$$

In practice, each current $g_{j}(j=1,2)$ is applied through one pair of electrodes attached at points $P_{j}, Q_{j} \in \partial \Omega$. Here, the points $P_{1}, P_{2}, Q_{1}$ and $Q_{2}$ are situated along the boundary $\partial \Omega$ in this order and separated by a distance greater than $2 \epsilon$. (See [7].) Hence we can assume, as in (1.2), the current $g_{j}$ is approximated by

$$
g_{j}(x)= \begin{cases}+\frac{I}{2 \epsilon} & \text { on }\left\{\left|x-P_{j}\right|<\epsilon\right\} \cap \partial \Omega \\ -\frac{I}{2 \epsilon} & \text { on }\left\{\left|x-Q_{j}\right|<\epsilon\right\} \cap \partial \Omega, \\ 0 & \text { otherwise, }\end{cases}
$$

where $I$ is the current sent to both electrodes at $P_{j}$ and $Q_{j}$, and $2 \epsilon$ is the width of each electrode. With these currents $g_{1}$ and $g_{2}$ as the Neumann data, from (2.5) we can easily see that the solution $\left(u_{1}, u_{2}\right) \in H^{1}(\Omega) \times H^{1}(\Omega)$ to the nonlinear system (4.1) satisfies

$$
\nabla u_{j}(x) \neq 0 \quad \text { for all } x \in \Omega, \quad j=1,2 .
$$

More generally, in this case we can prove that (4.2) holds as the following lemma. 
Lemma 4.1. Suppose that $\left(u_{1}, u_{2}\right) \in H^{1}(\Omega) \times H^{1}(\Omega)$ is a solution to the nonlinear system (4.1) with the Neumann data $g_{1}$ and $g_{2}$ defined in (4.3). Then we have

$$
\left|\nabla u_{1}(x) \times \nabla u_{2}(x)\right|>0 \quad \text { for all } x \in \Omega \text {. }
$$

Proof. To derive a contradiction, suppose that there exists a point $\xi \in \Omega$ such that

$$
\left|\nabla u_{1}(\xi) \times \nabla u_{2}(\xi)\right|=0 .
$$

Then there exists a nonzero vector $\left(c_{1}, c_{2}\right) \in \mathbb{R}^{2}$ so that $c_{1} \nabla u_{1}(\xi)+c_{2} \nabla u_{2}(\xi)=0$. Consider the function $w:=c_{1} u_{1}+c_{2} u_{2}$, which satisfies $\nabla w(\xi)=0$ and

$$
\begin{gathered}
\nabla \cdot\left(\frac{a_{1}}{\left|\nabla u_{1}\right|} \nabla w\right)=0 \quad \text { in } \Omega \\
\frac{a_{1}}{\left|\nabla u_{1}\right|} \frac{\partial w}{\partial \nu}=\tilde{g} \quad \text { on } \partial \Omega, \quad \text { and } \int_{\partial \Omega} w d s=0
\end{gathered}
$$

where $\tilde{g}=c_{1} g_{1}+c_{2} g_{2}$. By the assumption of

$$
\frac{a_{1}(x)}{\left|\nabla u_{1}(x)\right|}=\frac{a_{2}(x)}{\left|\nabla u_{2}(x)\right|} \in \Sigma
$$

we may regard $w$ as a solution to the classical Neumann problem (2.3) with the conductivity coefficient in the set $\Sigma$. Then all the properties in (2.4) hold for $w$.

On the other hand, the definition of $g_{j}$ in (4.3) yields

$$
\tilde{g}(x)= \begin{cases}+c_{1} \frac{I}{2 \epsilon} & \text { on }\left\{\left|x-P_{1}\right|<\epsilon\right\} \cap \partial \Omega, \\ +c_{2} \frac{I}{2 \epsilon} & \text { on }\left\{\left|x-P_{2}\right|<\epsilon\right\} \cap \partial \Omega \\ -c_{1} \frac{I}{2 \epsilon} & \text { on }\left\{\left|x-Q_{1}\right|<\epsilon\right\} \cap \partial \Omega \\ -c_{2} \frac{I}{2 \epsilon} & \text { on }\left\{\left|x-Q_{2}\right|<\epsilon\right\} \cap \partial \Omega \\ 0 & \text { otherwise. }\end{cases}
$$

Hence, by the ordering of the points $P_{1}, P_{2}, Q_{1}$ and $Q_{2}$, we easily see that for any nonzero vector $\left(c_{1}, c_{2}\right), \tilde{g} \neq 0$ and there exist two disjoint $\operatorname{arcs} \Gamma^{+}$and $\Gamma^{-}$contained in $\partial \Omega$ such that

$$
\Gamma^{+} \cup \Gamma^{-}=\partial \Omega, \quad \text { and } \quad \Gamma^{+} \subset\{\tilde{g} \geq 0\}, \quad \Gamma^{-} \subset\{\tilde{g} \leq 0\} .
$$

Therefore, it follows from (2.5) that $\nabla w(x) \neq 0$ for all $x \in \Omega$. In particular, $\nabla w(\xi) \neq 0$, hence it is a contradiction. This completes the proof.

For the sake of clarity, we will give in the following remark more detailed proof for the reason why the property (4.4) of nonzero $\tilde{g}$ implies $\nabla w \neq 0$ in $\Omega$, although it can be also found in $[1,2,13]$.

Remark 4.2. Suppose that $\nabla w(\xi)=0$, then by the maximum principle the level set $\{x \in \Omega \mid w(x)=w(\xi)\}$ divides $\Omega$ into more than four disjoint connected components $\Omega_{1}^{ \pm}, \cdots, \Omega_{m}^{ \pm}(m \geq 2)$ such that (see Figure 2)

$$
\bigcup_{k=1}^{m} \Omega_{k}^{+}=\{x \in \Omega \mid w(x)>w(\xi)\} \quad \text { and } \quad \bigcup_{k=1}^{m} \Omega_{k}^{-}=\{x \in \Omega \mid w(x)<w(\xi)\} .
$$

Applying the maximum principle again, the boundary of each component $\Omega_{k}^{ \pm}$ must occupy a portion $\gamma_{k}^{ \pm}$of $\partial \Omega$, that is, $\gamma_{k}^{ \pm}:=\partial \Omega_{k}^{ \pm} \cap \partial \Omega \neq \emptyset$ : If not, $\partial \Omega_{k}^{ \pm}$is a subset of the level curve $\{x \in \Omega \mid w(x)=w(\xi)\}$ and therefore by maximum principle $w$ is the constant equal to $w(\xi)$ in $\Omega_{k}^{ \pm}$. By the unique continuation, $\nabla w=0$ in the whole domain $\Omega$ and therefore $\tilde{g}=0$, which is a contradiction.

From the maximum-minimum principle

$$
\sup _{\Omega_{k}^{+}} w=\sup _{\partial \Omega_{k}^{+}} w=\sup _{\gamma_{k}^{+}} w \quad \text { and } \quad \inf _{\Omega_{k}^{-}} w=\inf _{\partial \Omega_{k}^{-}} w=\inf _{\gamma_{k}^{-}} w
$$




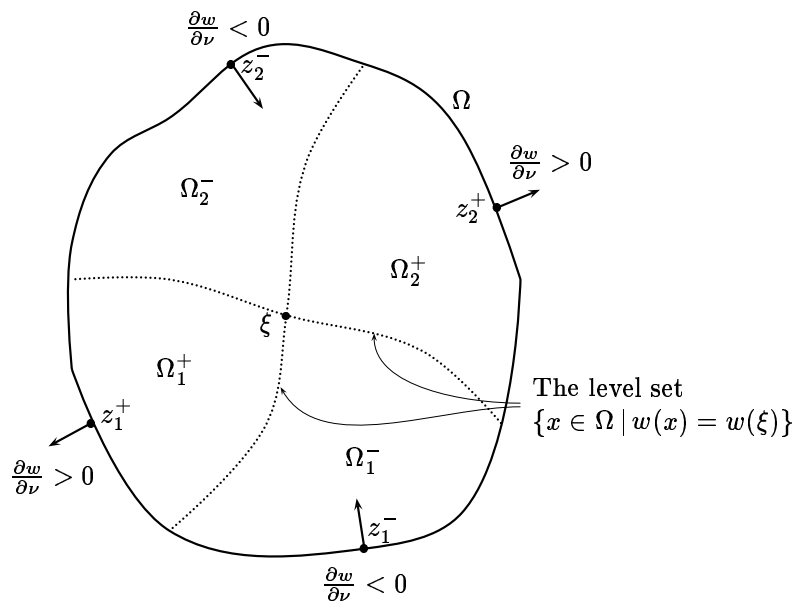

Figure 2. An illustration for Remark 4.2 when $m=2$.

there exist points $z_{k}^{+} \in \gamma_{k}^{+}$and $z_{k}^{-} \in \gamma_{k}^{-}$so that

$$
w\left(z_{k}^{+}\right)=\sup _{\Omega_{k}^{+}} w, \quad w\left(z_{k}^{-}\right)=\inf _{\Omega_{k}^{-}} w .
$$

By Hopf's lemma, we have $\tilde{g}\left(z_{k}^{+}\right)>0$ and $\tilde{g}\left(z_{k}^{-}\right)<0$ for $k=1, \cdots, m$. Since $m \geq 2$, $\tilde{g}$ cannot satisfy the property (4.4), which is a contradiction.

Lemma 4.1 tells us that two gradient vector fields $\nabla u_{1}$ and $\nabla u_{2}$ are neither vanishing nor parallel to each other at any points in $\Omega$. Based on this fact, we can prove the following uniqueness result for the inverse problem with two measurements.

Theorem 4.3. Suppose that $\left(u_{1}, u_{2}\right),\left(\tilde{u}_{1}, \tilde{u}_{2}\right) \in H^{1}(\Omega) \times H^{1}(\Omega)$ are solutions to the nonlinear system (4.1) with the Neumann data $g_{1}$ and $g_{2}$ defined in (4.3). Then the edge of the conductivity image is uniquely determined by $\left(a_{1}, a_{2}\right)$ in such a way that

$\left\{x \in \Omega \mid \frac{a_{j}}{\left|\nabla u_{j}\right|}\right.$ is discontinuous at $\left.x\right\}=\left\{x \in \Omega \mid \frac{a_{j}}{\left|\nabla \tilde{u}_{j}\right|}\right.$ is discontinuous at $\left.x\right\}$.

Proof. Since $\left(u_{1}, u_{2}\right)$ satisfies

$$
\frac{a_{1}}{\left|\nabla u_{1}\right|}=\frac{a_{2}}{\left|\nabla u_{2}\right|} \in \Sigma,
$$

there exist $\sigma_{0} \in \mathcal{C}^{\alpha}(\bar{\Omega})$ and $\left\{\left(\sigma_{k}, D_{k}\right) \mid \sigma_{k} \in \mathcal{C}^{\alpha}\left(\bar{D}_{k}\right), \bar{D}_{k} \subset \Omega\right\}_{k=1}^{M}$ for some $M \in \mathbb{N}$, which satisfy

$$
\frac{a_{j}}{\left|\nabla u_{j}\right|}=\sigma_{0}+\sum_{k=1}^{M} \sigma_{k} \chi_{D_{k}} \in \Sigma \text {. }
$$

Hence, from (2.1) we have

$$
\sigma:=\sigma_{0}+\sum_{k=1}^{M} \sigma_{k} \chi_{D_{k}} \in \mathcal{C}^{\alpha}\left(\cup_{k=1}^{M} \bar{D}_{k}\right) \cap \mathcal{C}^{\alpha}\left(\Omega \backslash \cup_{k=1}^{M} D_{k}\right),
$$

and $u_{j}$ can be viewed as a solution of (2.3) when $g$ is substituted by $g_{j}$. Thus, from (b) in (2.4) we get

$$
\nabla u_{j} \in \mathcal{C}^{\alpha}\left(\cup_{k=1}^{M} \bar{D}_{k}\right) \cap \mathcal{C}^{\alpha}\left(\Omega \backslash \cup_{k=1}^{M} D_{k}\right) .
$$

From (4.5), we have $a_{j}(x)=\sigma(x)\left|\nabla u_{j}(x)\right|$, which implies that

$$
a_{j} \in \mathcal{C}^{\alpha}\left(\cup_{k=1}^{M} \bar{D}_{k}\right) \cap \mathcal{C}^{\alpha}\left(\Omega \backslash \cup_{k=1}^{M} D_{k}\right) \quad \text { for } j=1,2,
$$


by the aid of (4.6) and (4.7). Therefore, we get

$$
A:=\left\{x \in \Omega \mid a_{1} \text { or } a_{2} \text { is discontinuous at } x\right\} \subset \bigcup_{k=1}^{M} \partial D_{k} .
$$

For converse of (4.8), fix any $\xi \in \partial D_{k}$ for any $k=1, \cdots, M$. It follows from Lemma 4.1 that either

$$
\frac{\partial u_{1}^{+}}{\partial \tau}(\xi) \neq 0 \quad \text { or } \quad \frac{\partial u_{2}^{+}}{\partial \tau}(\xi) \neq 0
$$

where $u_{j}^{+}:=\left.u_{j}\right|_{\Omega \backslash \bar{D}_{k}}$ for $j=1,2$, and $\partial / \partial \tau$ denotes the tangential derivative on $\partial D_{k}$. By the properties $(c)$ and $(d)$ in (2.4), we get

$$
\sigma_{0}(\xi) \frac{\partial u_{j}^{+}}{\partial \nu}(\xi)=\left(\sigma_{0}(\xi)+\sigma_{k}(\xi)\right) \frac{\partial u_{j}^{-}}{\partial \nu}(\xi) \quad \text { and } \quad \frac{\partial u_{j}^{+}}{\partial \tau}(\xi)=\frac{\partial u_{j}^{-}}{\partial \tau}(\xi),
$$

where $u_{j}^{-}:=\left.u_{j}\right|_{D_{k}}$ and $\nu$ denotes the outward unit normal to $\partial D_{k}$. Considering $a_{j}=\sigma\left|\nabla u_{j}\right|$, a simple calculation yields that

$$
\left|a_{j}^{-}(\xi)\right|^{2}=\left|a_{j}^{+}(\xi)\right|^{2}+\left(\left(\sigma_{0}(\xi)+\sigma_{k}(\xi)\right)^{2}-\left(\sigma_{0}(\xi)\right)^{2}\right)\left|\frac{\partial u_{j}^{+}}{\partial \tau}(\xi)\right|^{2},
$$

where $a_{j}^{-}:=\left.a_{j}\right|_{D_{k}}$ and $a_{j}^{+}:=\left.a_{j}\right|_{\Omega \backslash \bar{D}_{k}}$. Since $\sigma_{k}(\xi) \neq 0$ by definition of $\Sigma$, by the aid of (4.9) the second term on the right hand side of (4.10) is nonzero for either $j=1$ or $j=2$. Thus we show that $a_{1}$ or $a_{2}$ is discontinuous at $\xi$, and so $\xi \in A$. This proves that $\cup_{k=1}^{M} \partial D_{k} \subset A$. Hence, from (4.8) we conclude that $\cup_{k=1}^{M} \partial D_{k}=A$.

On the other hand, from (4.5) and (2.2), we can easily see that

$$
\left\{x \in \Omega \mid \frac{a_{j}}{\left|\nabla u_{j}\right|} \text { is discontinuous at } x\right\}=\bigcup_{k=1}^{M} \partial D_{k}=A .
$$

Because we have used only the fact that $\left(u_{1}, u_{2}\right)$ is a solution to the nonlinear system (4.1), we can derive the same conclusion as (4.11) for $\left(\tilde{u}_{1}, \tilde{u}_{2}\right)$

$$
\left\{x \in \Omega \mid \frac{a_{j}}{\left|\nabla \tilde{u}_{j}\right|} \text { is discontinuous at } x\right\}=\bigcup_{k=1}^{\tilde{M}} \partial \tilde{D}_{k}=A,
$$

for some mutually disjoint domains $\tilde{D}_{k} \subset \Omega$. Since the set $A$ is completely determined by the data $\left(a_{1}, a_{2}\right)$, the proof is completed by (4.11) and (4.12).

Theorem 4.3 shows that the region where the conductivity distribution has jumps can be uniquely detected by the observation of discontinuities of the measured data $\left(a_{1}, a_{2}\right)$. In the following theorem, we show that the conductivity values as well as the unknown inclusions can be determined in a simple case when the conductivity distribution $\sigma \in \Sigma$ is known to be piecewise constants.

Theorem 4.4. Suppose that $\left(u_{1}, u_{2}\right),\left(\tilde{u}_{1}, \tilde{u}_{2}\right) \in H^{1}(\Omega) \times H^{1}(\Omega)$ are solutions to the nonlinear system (4.1) with the Neumann data $g_{1}$ and $g_{2}$ defined in (4.3). Suppose that $\frac{a_{j}}{\left|\nabla u_{j}\right|}$ and $\frac{a_{j}}{\left|\nabla \tilde{u}_{j}\right|}$ are piecewise constants, that is,

$$
\frac{a_{j}}{\left|\nabla u_{j}\right|}=1+\sum_{k=1}^{M} \mu_{k} \chi_{D_{k}} \quad \text { and } \quad \frac{a_{j}}{\left|\nabla \tilde{u}_{j}\right|}=1+\sum_{k=1}^{\tilde{M}} \tilde{\mu}_{k} \chi_{\tilde{D}_{k}},
$$

where $\mu_{k}, \tilde{\mu}_{k}$ are nonzero constants satisfying $-1<\mu_{k}, \tilde{\mu}_{k}<\infty$. Then $\left(u_{1}, u_{2}\right)$ and $\left(\tilde{u}_{1}, \tilde{u}_{2}\right)$ are the same. 
Proof. First, we will prove that

$$
\frac{a_{j}}{\left|\nabla u_{j}\right|}=\frac{a_{j}}{\left|\nabla \tilde{u}_{j}\right|}
$$

From (4.13), and (4.11), (4.12) in the proof of Theorem 4.3, the edge of the conductivity image is uniquely determined, that is, $M=\tilde{M}$ and $\bigcup_{k=1}^{M} D_{k}=\bigcup_{k=1}^{\tilde{M}} \tilde{D}_{k}$. Thus, for (4.14) it only remains to prove that $\mu_{k}=\tilde{\mu}_{k}$ for $k=1, \cdots, M$. For this, it suffices to show that $\mu_{k}$ can be uniquely determined by the measured data $\left(a_{1}, a_{2}\right)$ analogously as explained in the proof of Theorem 4.3. To be precise, $\mu_{k}$ will be shown to be determined by

$$
\mu_{k}=\sqrt{1+m_{k}}-1, \quad k=1, \cdots, M,
$$

where the number $m_{k}$ is defined by

$$
m_{k}:= \begin{cases}\max _{\xi \in \partial D_{k}}\left\{\left|\frac{a_{1}^{-}(\xi)}{a_{1}^{+}(\xi)}\right|^{2}-1\right\}, & \text { if } a_{1}^{-} \geq a_{1}^{+} \text {on } \partial D_{k}, \\ \min _{\xi \in \partial D_{k}}\left\{\left|\frac{a_{1}^{-}(\xi)}{a_{1}^{+}(\xi)}\right|^{2}-1\right\}, & \text { if } a_{1}^{-} \leq a_{1}^{+} \text {on } \partial D_{k} .\end{cases}
$$

Here, $a_{1}^{-}:=\left.a_{1}\right|_{D_{k}}$ and $a_{1}^{+}:=\left.a_{1}\right|_{\Omega \backslash \overline{D_{k}}}$.

From (4.13), we have $a_{1}^{+}=\left|\nabla u_{1}^{+}\right|$on $\partial D_{k}$, thus it follows

$$
\left|\frac{a_{1}^{-}(\xi)}{a_{1}^{+}(\xi)}\right|^{2}-1=\frac{\left|a_{1}^{-}(\xi)\right|^{2}-\left|a_{1}^{+}(\xi)\right|^{2}}{\left|\nabla u_{1}^{+}(\xi)\right|^{2}}, \quad \xi \in \partial D_{k} .
$$

By the aid of (4.10) (in our case, $\sigma_{0}(\xi)=1$ and $\sigma_{k}(\xi)=\mu_{k}$ ), we easily observe that either $a_{1}^{-} \geq a_{1}^{+}$or $a_{1}^{-} \leq a_{1}^{+}$on $\partial D_{k}$. In case when $a_{1}^{-} \geq a_{1}^{+}$, from (4.17) and (4.10) we have

$$
\left|\frac{a_{1}^{-}(\xi)}{a_{1}^{+}(\xi)}\right|^{2}-1 \leq \frac{\left|a_{1}^{-}(\xi)\right|^{2}-\left|a_{1}^{+}(\xi)\right|^{2}}{\left|\partial u_{1}^{+} / \partial \tau(\xi)\right|^{2}}=\mu_{k}\left(\mu_{k}+2\right),
$$

for all $\xi \in \partial D_{k}$. In case when $a_{1}^{-} \leq a_{1}^{+}$, we get the similar result given by

$$
\left|\frac{a_{1}^{-}(\xi)}{a_{1}^{+}(\xi)}\right|^{2}-1 \geq \mu_{k}\left(\mu_{k}+2\right) \text {. }
$$

Now we will find the optimizer $z \in \partial D_{k}$ of (4.16). Applying the divergence theorem on $\Omega \backslash \bar{D}_{k}$, we get

$$
0=\int_{\partial \Omega} \frac{a_{1}}{\left|\nabla u_{1}\right|} \frac{\partial u_{1}}{\partial \nu} d s-\int_{\partial D_{k}} \frac{\partial u_{1}^{+}}{\partial \nu} d s=-\int_{\partial D_{k}} \frac{\partial u_{1}^{+}}{\partial \nu} d s
$$

noting that $u_{1}$ belongs to $\mathcal{C}^{1, \alpha}\left(\Omega \backslash \cup_{k=1}^{M} D_{k}\right)$ from (2.4) and $a_{1}^{+} /\left|\nabla u_{1}^{+}\right|=1$ on $\partial D_{k}$. Hence there exists a point $z \in \partial D_{k}$ satisfying $\partial u_{1}^{+} / \partial \nu(z)=0$, and by Lemma 4.1 we have

$$
\left|\frac{\partial u_{1}^{+}}{\partial \tau}(z)\right|=\left|\nabla u_{1}^{+}(z)\right|>0 .
$$

From (4.17), (4.18), and the jump relation (4.10), we obtain

$$
\left|\frac{a_{1}^{-}(z)}{a_{1}^{+}(z)}\right|^{2}-1=\mu_{k}\left(\mu_{k}+2\right)
$$

which implies that the point $z \in \partial D_{k}$ is the optimizer of (4.16). Thus it is clear that the number $m_{k}$ defined in (4.16) is given by $m_{k}=\mu_{k}\left(\mu_{k}+2\right)>-1$ because $\mu_{k}>-1$. Therefore we conclude that $\mu_{k}=\sqrt{1+m_{k}}-1$, which proves (4.15), and hence (4.14). 
Finally, from (4.13) and (4.14) we see that $u_{j}$ and $\tilde{u}_{j}$ can be viewed as the solutions of (2.3) when $g$ is substituted by $g_{j}$ and $\sigma:=1+\sum_{k=1}^{M} \mu_{k} \chi_{D_{k}}$, since both $\left(u_{1}, u_{2}\right)$ and $\left(\tilde{u}_{1}, \tilde{u}_{2}\right)$ are solutions to the nonlinear system (4.1). Hence by the uniqueness of the classical Neumann problem (2.3), we verify that $u_{1}=\tilde{u}_{1}$ and $u_{2}=\tilde{u}_{2}$, which completes the proof.

\section{Conclusion and Numerical examples}

A new reconstruction algorithm, so called $J$-substitution algorithm, was presented in [7] without uniqueness proofs to provide an impressively high resolution conductivity image $\sigma$ in simulations based on internal current density a obtained from MRI system. For this algorithm, two different internal current densities $a_{1}$ and $a_{2}$ induced by two different applied currents $g_{1}$ and $g_{2}$ defined in (4.3) were used. In this paper, Theorem 4.3 has proved the uniqueness of the edge detection for piecewise continuous conductivities, and Theorem 4.4 has shown that a piecewise constant conductivity distribution can be completely reconstructed from $a_{1}$ and $a_{2}$.

On the other hand, it is worth investigating whether one could recover the conductivity distribution with only one internal current density, which means equivalently whether the nonlinear Neumann boundary value problem (3.2) could be solved uniquely. Theorem 3.1 has given a negative answer to this question.

In this section, we will present a numerically obtained example of non-uniqueness with one measurement which has been discussed in section 3. Suppose that Figure 3 represents an internal current density $a(x)$ on a cross-section $\Omega=(-1,1) \times(-1,1)$ of the human body induced by the applied the current

$$
g(x)= \begin{cases}1 & \text { if } x_{1}=1 \\ -1 & \text { if } x_{1}=-1 \\ 0 & \text { if otherwise }\end{cases}
$$

which can be viewed as an electrode attachment model in $(1.2)$ when $P=(1,0)$, $Q=(-1,0), I=2$, and $\epsilon=1$. We have numerically obtained this current density

$$
a(x):=\sigma(x)|\nabla u(x)|
$$

by assuming a conductivity distribution $\sigma$ (in our experiment, $\sigma$ is assumed to be $\sigma_{1}$ in Figure 5) and numerically solving the classical Neumann problem (2.3) with Neumann data $g$ in $(5.1)$ to calculate $|\nabla u(x)|$. As a numerical solver for (2.3),

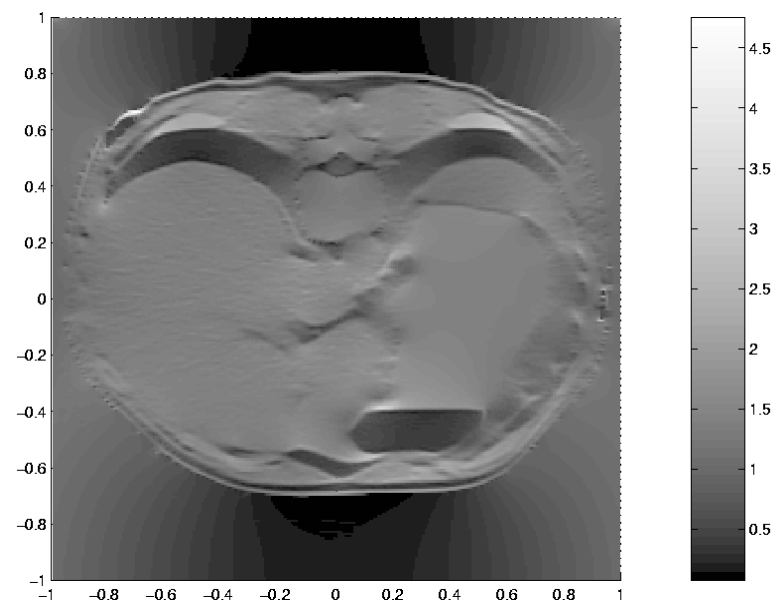

FIGURE 3. Simulated current density $a(x)$. 


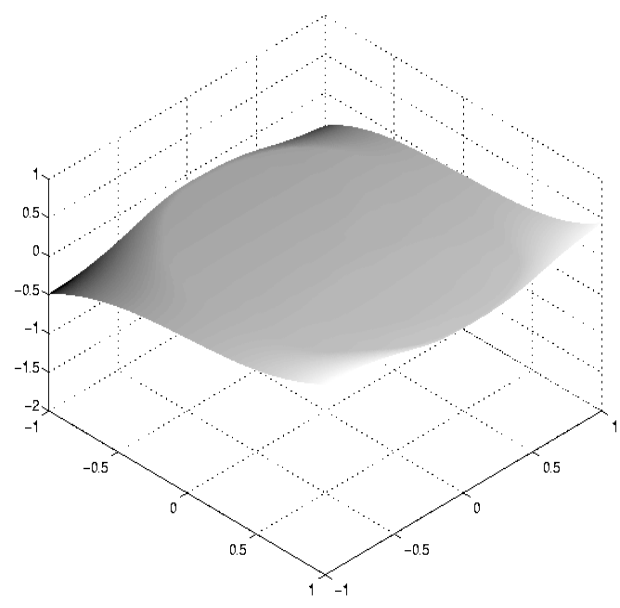

$u_{1}(x)$

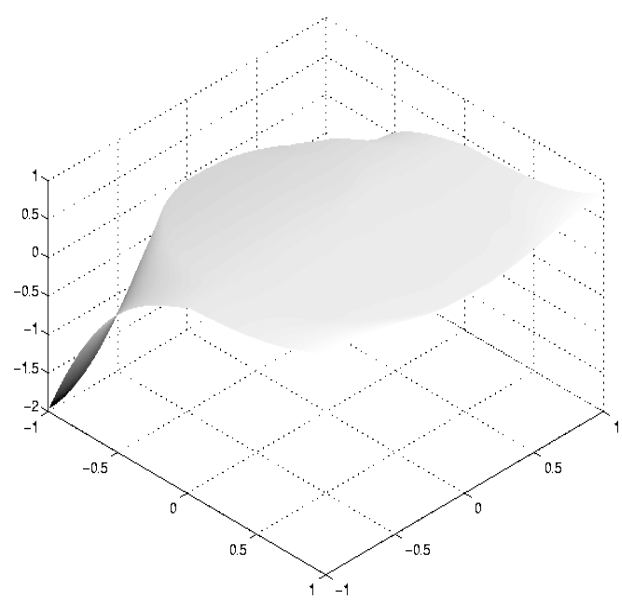

$u_{2}(x)$

FIGURE 4. Two different solutions $u_{1}$ and $u_{2}$ to the problem (3.2).

we have adopted the cell-centered finite difference scheme explained in [7]. In real situation, the current density $a(x)$ is provided by a suitable MRI experiment called current density imaging $[4,6,10,11,12,15]$.

With this $a$ and $g$, we can construct infinitely many solutions of the nonlinear Neumann boundary value problem (3.2) by virtue of Theorem 3.1. Here we present two different solutions $u_{1}$ and $u_{2}$, respectively given in Figure 4 . Indeed, $u_{1}$ is equal to $u$ that has been used to generate the simulated current density $a$ in (5.2), and $u_{2}$ corresponds to $u_{t, \lambda}$ defined in the proof of Theorem 3.1 in case when $t=0$ and $\lambda=5$. These two different solutions yield two distinct conductivity images

$$
\sigma_{1}(x)=\frac{a(x)}{\left|\nabla u_{1}(x)\right|} \quad \text { and } \quad \sigma_{2}(x)=\frac{a(x)}{\left|\nabla u_{2}(x)\right|},
$$

which are respectively shown in Figure 5. Hence, we conclude that only one internal current density information is insufficient for the unique determination of conductivity distributions.
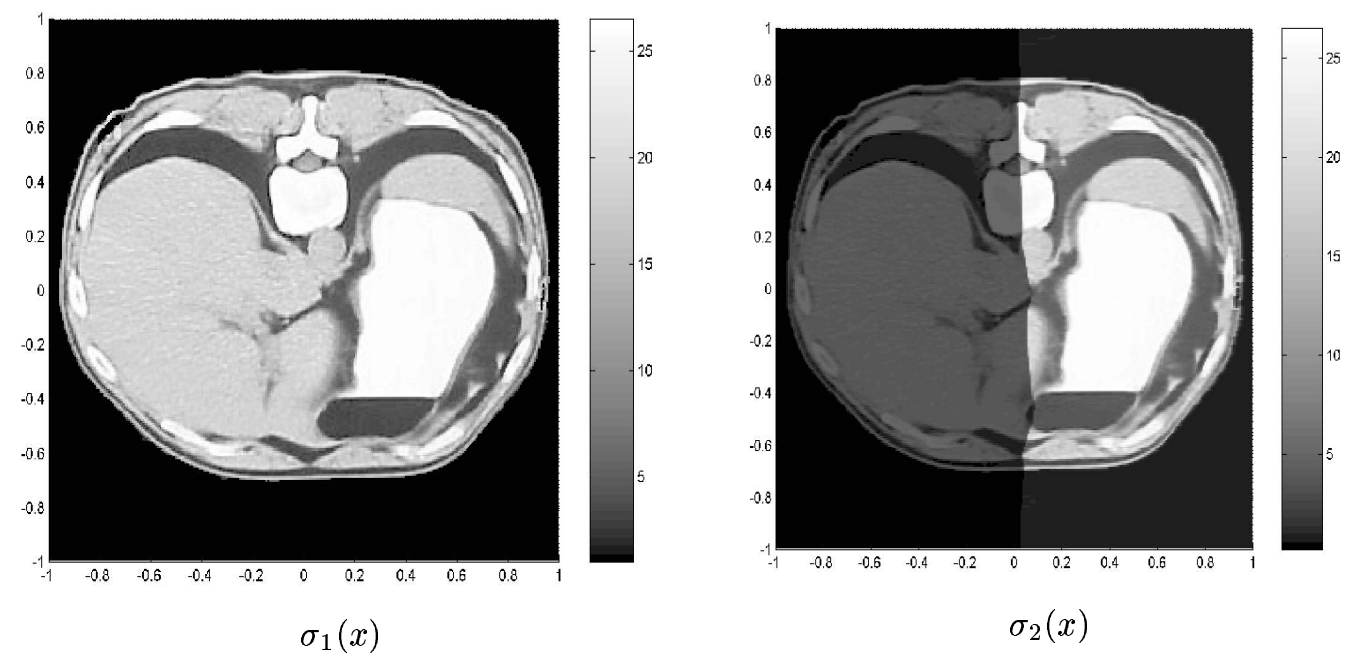

FiguRE 5. Two distinct conductivity images generated by $u_{1}$ and $u_{2}$. 


\section{REFERENCES}

[1] G. Aless andrini, V. Isakov, and J. Powell, Local uniqueness in the inverse problem with one measurement, Trans. Amer. Math. Soc., 347 (1995), pp. 3031-3041.

[2] G. Alessandrini And R. Magnanini, The index of isolated critical points and solutions of elliptic equations in the plane, Ann. Scuola Norm. Sup. Pisa Cl. Sci. (4), 19 (1992), pp. 567589.

[3] K.-S. Cheng, D. Isaacson, J. C. Newell, and D. G. Gisser, Electrode model for electric current computed tomography, IEEE Trans. Biomed. Eng., 36 (1989), pp. 918-924.

[4] H. R. Gamba And D. T. Delpy, Measurement of electrical current density distribution within the tissues of the head by magnetic resonance imaging, Med. Biol. Eng. Comp., 36 (1998), pp. $165-170$.

[5] D. Gilbarg and N. S. Trudinger, Elliptic Partial Differential Equations of Second Order, Springer-Verlag, Berlin, 1983.

[6] Y. Z. Ider AND L. T. Muftuler, Measurement of ac magnetic field distribution using magnetic resonance imaging, IEEE Trans. Med. Imag., 16 (1997), pp. 617-622.

[7] O. Kwon, E. Woo, J. Yoon, And J. K. Seo, Magnetic Resonance Electrical Impedance Tomography (MREIT) : Simulation Study of J-Substitution Algorithm, submitted for publication.

[8] O. A. Ladyzhenskaya and N. N. Ural'Ceva, Linear and Quasilinear Equations of Elliptic Type, Izdat. "Nauka", Moscow, pp. 576, 1973.

[9] J. L. Mueller, D. Isaacson, And J. C. Newell, A reconstruction algorithm for electrical impedance tomography data collected on rectangular electrode array, IEEE Trans. Biomed. Eng., 46 (1999), pp. 1379-1386.

[10] G. C. Scott, M. L. G. Joy, R. L. Armstrong, and R. M. Henkelman, Measurement of nonuniform current density by magnetic resonance, IEEE Trans. Med. Imag., 10 (1991), pp. $362-374$.

[11] G. C. Scott, M. L. G. Joy, R. L. Armstrong, and R. M. Henkelman, Sensitivity of magnetic-resonance current density imaging, J. Mag. Res., 97 (1992), pp. 235-254.

[12] G. C. Scott, M. L. G. Joy, R. L. Armstrong, and R. M. Henkelman, Electromagnetic considerations for RF current density imaging, IEEE Trans. Med. Imag., 14 (1995), pp. 515524.

[13] J. K. SEO, A uniqueness result on inverse conductivity problem with two measurements, J. Fourier Anal. Appl., 2 (1996), pp. 227-235.

[14] J. G. WeBster, ED., Electrical Impedance Tomography, Adam Hilger, Bristol, 1990.

[15] E. J. Woo, S. Y. LeE, AND C. W. Mun, Impedance tomography using internal current density distribution measured by nuclear magnetic resonance, Proc. SPIE, 2299 (1994), pp. 377-385.

E-mail address: oikwon@math.snu.ac.kr

E-mail address: seoj@yonsei.ac.kr

E-mail address: jryoon@kias.re.kr 